\title{
Background Factors Associated with the Complications of Coronary Artery Lesions Caused by Kawasaki Disease
}

\author{
Toshimasa Nakada \\ Department of Pediatrics, Aomori Prefectural Central Hospital, Aomori City, Japan
}

Email address:

toshimasanakada@yahoo.co.jp

\section{To cite this article:}

Toshimasa Nakada. Background Factors Associated with the Complications of Coronary Artery Lesions Caused by Kawasaki Disease. Clinical Medicine Research. Vol. 4, No. 5, 2015, pp. 127-131. doi: 10.11648/j.cmr.20150405.11

\begin{abstract}
Appropriate therapy during the acute phase of Kawasaki disease to prevent large coronary artery lesions (CAL) has not been established. The aim of this retrospective study was to investigate the usefulness of an initial single intravenous immunoglobulin (IVIG) therapy. In this study, I included a total of 200 pediatric patients who had received $2 \mathrm{~g} / \mathrm{kg} / \mathrm{day}$ IVIG therapy for Kawasaki disease between 1999 and 2015 at the Department of Pediatrics, Aomori Prefectural Central Hospital. An initial IVIG therapy starting on day 5 was used as first-line therapy when possible. The second-line therapy was additional IVIG therapy, and the third-line therapy was an urinastatin infusion or plasma exchange. All patients received an initial single IVIG therapy with delayed or with concomitant administration of aspirin or flurbiprofen. Initial IVIG therapy resistance occurred in 48 of 200 patients (24\%), and 17 patients (9\%) received additional IVIG therapy. Four patients received urinastatin and one patient received plasma exchange as the third-line therapy. Before the 30th day, the prevalence of CAL was $5 \%$ (10/200); after 30 days, it was $2 \%(4 / 200)$. The maximal internal CAL diameter was $4.8 \mathrm{~mm}(\mathrm{Z}$ score $=6.3)$ among all patients. Variable factors including IVIG resistance, responsiveness, and relapse of disease were associated with CAL complications. An initial single IVIG therapy may be useful for the prevention of large CAL caused by different factors of Kawasaki disease.
\end{abstract}

Keywords: Kawasaki Disease, Intravenous Immunoglobulin Therapy, Coronary Artery Lesions, Anti-inflammatory Drugs, Aspirin, Flurbiprofen

\section{Introduction}

Kawasaki disease is an acute systemic vasculitis of unknown cause that affects mainly infants and children [1]. Coronary artery lesions $(\mathrm{CAL})$ are one of the most important complications of this disease. During the acute phase (before day 30 ), coronary artery aneurysms develop. During the convalescent phase (after day 30), large aneurysms develop into subsequent stenosis and these stenotic lesions cause myocardial ischemia. On the other hand, small aneurysms regress without leaving stenotic lesions.

Long-term follow-up studies have shown that a maximum CAL size $>5 \mathrm{~mm}$ was a statistically significant predictive risk factor for myocardial ischemia, and that all CAL $\leq 5 \mathrm{~mm}$ in size regressed to normal size [2]. Another study reported that the threshold diameter for acute phase CAL that developed into subsequent stenosis was $6.0 \mathrm{~mm}$ [3]. Therefore, the prevention of CAL of $>5 \mathrm{~mm}$ may be an important goal in the acute treatment of Kawasaki disease to prevent coronary artery stenosis in later stages of the disease [4].
Treatment with intravenous immunoglobulin (IVIG) therapy reduces the occurrence of CAL caused by Kawasaki disease $[5,6]$. The current standard therapy during the acute phase of Kawasaki disease is $2 \mathrm{~g} / \mathrm{kg}$ /day IVIG therapy [7]. Combination regimens of IVIG and other drugs including steroids and infliximab have been tried as the initial therapy for patients with Kawasaki disease [8,9]. However, the treatment for the prevention of large CAL has not been established, and not enough studies have been performed with regard to initial IVIG monotherapy in spite of the safety and effectiveness of this therapy [4].

The background factors associated with the development of CAL among patients who had received $2 \mathrm{~g} / \mathrm{kg}$ /day IVIG therapy remain unclear. A previous epidemiological study revealed that CAL may occur in both immunoglobulinresistant and -responsive patients [10]. Clarification of these background factors may lead to appropriate acute phase treatment for CAL suppression.

A recent study showed that an initial single IVIG therapy with delayed administration of aspirin or flurbiprofen was effective for the suppression of CAL caused by Kawasaki 
disease [11]. The hypothesis of this study was that the background factors associated with CAL development are variable and that an initial single IVIG therapy may be useful in the CAL suppression. Accordingly, this study investigated the background factors associated with the development of CAL and outcome of CAL who had received an initial $2 \mathrm{~g} / \mathrm{kg} /$ day IVIG therapy for Kawasaki disease.

I excluded the patients who received $1 \mathrm{~g} / \mathrm{kg} /$ day IVIG therapy and those who associated with CAL before the start of therapy because this study aimed to investigate the usefulness of $2 \mathrm{~g} / \mathrm{kg} /$ day IVIG therapy for prevention of CAL. Recent study revealed that the regimen using $1 \mathrm{~g} / \mathrm{kg} /$ day IVIG therapy may be safe and useful for the patients associated with CAL before the start of therapy [12].

Previous studies showed that the prevalence of CAL were high among the patients with recurrent Kawasaki disease [13, 14]. Therefore, I excluded the patients with recurrent Kawasaki disease to exclude this bias.

\section{Methods}

This retrospective study included 200 consecutive patients who had received an initial $2 \mathrm{~g} / \mathrm{kg}$ /day IVIG therapy for Kawasaki disease between January 1999 and February 2015 at the Department of Pediatrics, Aomori Prefectural Central Hospital. The diagnosis of Kawasaki disease was based on the Japanese criteria (Fifth edition) [15]. 9 patients with disease recurrence, 4 patients associated with CAL before the start of therapy, and 6 patients who received $1 \mathrm{~g} / \mathrm{kg} /$ day IVIG therapy during the study period were excluded.

In this study, recurrence and relapse were defined differently. When Kawasaki disease recurred after the initial disappearance of the major symptoms and improvement in the test results, it was defined as a recurrence. If a patient became afebrile during the acute phase, an exacerbation or reappearance of major symptoms without other pyrogenic disease was defined as a relapse.

The participants were divided into $\mathrm{S}$ and $\mathrm{T}$ groups. The $\mathrm{S}$ group included 134 patients who had received an initial single IVIG therapy with the delayed administration of aspirin or flurbiprofen, whereas the $\mathrm{T}$ group included 66 patients who had received these anti-inflammatory drugs concomitantly with IVIG therapy. In the S group, the antiinflammatory drugs were initiated within $24 \mathrm{~h}$ after the end of the initial IVIG therapy. In this study, an initial single IVIG therapy was the regimen used to treat the patients in both the $\mathrm{S}$ and $\mathrm{T}$ groups.

\subsection{Anti-inflammatory Drugs Therapy}

The choice between aspirin and flurbiprofen was made by each doctor after considering the patient's liver function and the risk of Reye syndrome at the influenza pandemic. Flurbiprofen was more commonly used before 2009. Aspirin was initiated at a dose of $30 \mathrm{mg} / \mathrm{kg} /$ day and decreased to 5-10 $\mathrm{mg} / \mathrm{kg} /$ day when the patients became afebrile. Flurbiprofen was initiated at a dose of $3-5 \mathrm{mg} / \mathrm{kg} / \mathrm{day}$ and decreased to $3 \mathrm{mg} / \mathrm{kg} /$ day when the patient became afebrile.
The regimen that was prescribed for the $S$ group was not used until after 2004. Some patients had received the S group therapy regimen between 2004 and 2008. After 2009, the S group regimen was used for all patients.

\subsection{IVIG Therapy}

During the study period, an initial IVIG regimen of 2 $\mathrm{g} / \mathrm{kg} /$ day starting on the fifth day of the illness was used as the first-line therapy when possible. The indication for additional therapy in resistant patients was determined between 48 and $72 \mathrm{~h}$ after the end of the initial IVIG therapy. The diagnosis was made according to clinical parameters, including body temperature, major signs of Kawasaki disease, general condition, and laboratory data. The second-line therapy was additional IVIG therapy, and the third-line therapy was an urinastatin infusion. Plasma exchange was adopted after 2014 as another third-line therapy option. Written informed consent was obtained from the parents or guardians of all the patients before the initial therapy.

Patients with a response to IVIG therapy were defined as those patients who became afebrile (temperature $<37.5{ }^{\circ} \mathrm{C}$ for $24 \mathrm{~h}$ ) within $24 \mathrm{~h}$ after the completion of the initial IVIG infusion. IVIG-resistant patients were defined as those not meeting these criteria.

\subsection{Diagnosis of CAL}

Coronary artery lesions were diagnosed by echocardiography according to the Japan Ministry of Health and Welfare criteria [16]. CAL was defined as an artery diameter that exceeded $3 \mathrm{~mm}$ in a child below 5 years of age or a diameter that exceeded $4 \mathrm{~mm}$ in a child aged 5 years or older. Transient CAL was defined as the disappearance of CAL within 30 days of the illness. In this study, a CAL that was larger than $5 \mathrm{~mm}$ or larger than a $\mathrm{Z}$ score of 6.5 was defined as a large CAL.

\subsection{Statistical Analysis}

The statistical analyses were performed with StatFlex ${ }^{\circledR}$ Ver. 6 for Windows (Artech Co., Ltd. Osaka, Japan). The chisquare test, Fisher's exact test, and Mann-Whitney U test were used as appropriate. A P value of $<0.05$ was considered statistically significant.

\section{Results}

200 patients were 105 boys and 95 girls. The mean age was 2 years, 8 months (age range: 2 months to 13 years, 3 months).

Aspirin and flurbiprofen were administered in 91 and 109 patients, respectively. The prevalence of aspirin/flurbiprofen in the $\mathrm{S}$ and $\mathrm{T}$ groups was 74/60 and 17/49 $(\mathrm{P}<0.001)$, respectively. The male / female ratio between the $\mathrm{S}$ vs. T group were 65 / 69 vs. 40 / $26(\mathrm{P}=0.107)$. The mean age and age range between the $\mathrm{S}$ vs. T group were 2 years, 10 months (age range: 2 months to 13 years, 3 months) vs. 2 years, 5 months (age range: 3 months to 9 years, 6 months $)(P=0.512)$. 
The median start time of the initial IVIG therapy was the fifth day of illness (range: day 3-16 of illness). Initial IVIG therapy resistance occurred in 48 of the 200 patients (24\%); 17 patients $(9 \%)$ received additional IVIG: 13 patients for initial IVIG resistance and 4 patients for relapse, respectively. Four patients received urinastatin and one patient received a plasma exchange as a third-line therapy. Among the patients that received a third-line therapy, one patient received steroids after the urinastatin infusion because of prolonged fever and intractable arthralgia, and another patient received IVIG therapy as a fourth-line therapy after the plasma exchange. One patient who received steroids complicated no CAL.

The prevalence of CAL before day 30 was 5\% (10/200); after 30 days, it was $2 \%(4 / 200)$. The prevalence of CAL before and after 30 days of illness between the $\mathrm{S}$ and $\mathrm{T}$ groups was $2 / 134$ and $8 / 66(\mathrm{P}=0.003)$ and $1 / 134$ and $3 / 66(\mathrm{P}$ $=0.106)$, respectively. The maximal internal CAL diameters were $4.8 \mathrm{~mm}(Z$ score $=6.3)$ among all patients. The patient with the largest CAL diameter (Patient 1 of Fig. 1, Table 1 and 2) had CAL on day 8 , and she received a plasma exchange on day 9 at the hospital of Hirosaki University School of Medicine for 3 days. Her CAL diameter was 4.8 $\mathrm{mm}$ on day 21 of her illness. However, echocardiography on day 52 of illness showed the regression of CAL and a normal internal coronary artery size.

The four patients that complicated CAL after 30 days of illness were evaluated using selective coronary arteriography at a median of 8 months (range: 6-16 months) after disease onset. The coronary arteriograms of all four patients revealed that all CAL had regressed without leaving stenotic lesions.

Figure 1 shows the clinical courses after an initial single $2 \mathrm{~g} / \mathrm{kg} /$ day IVIG therapy. The prevalence of CAL after 30 days (CCAL) in resistant patients compared with responders was $3 / 48$ vs. $1 / 152(\mathrm{P}=0.044)$. Patient 3 developed complicated CCAL after relapse.

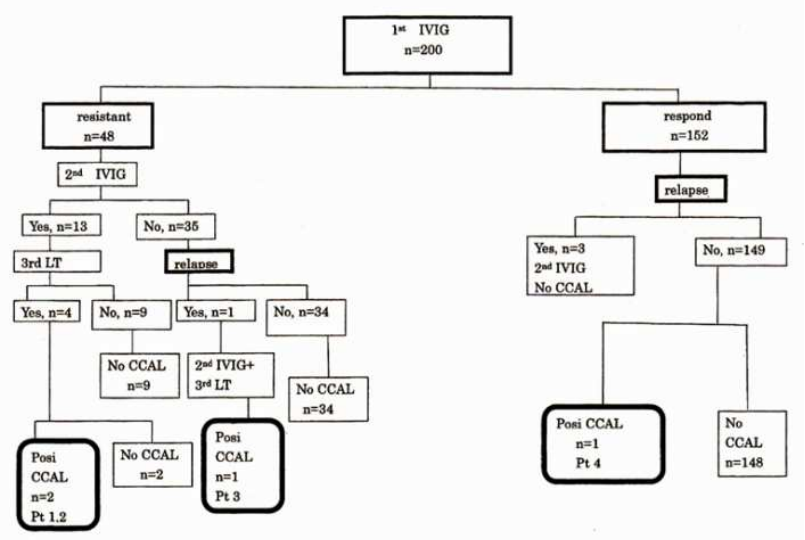

IVIG: intravenous immunoglobulin, 3rd LT: 3rd line therapy, CCAL: coronary artery lesions after 30 days of illness, Posi CCAL: patients with CCAL, No CCAL: patients without CCAL, Pt: patients.

Figure 1. Clinical courses after initial single $2 \mathrm{~g} / \mathrm{kg} /$ day IVIG therapy.

Table 1 shows the clinical features of the four patients that developed CCAL. Three of the four patients had received concomitant anti-inflammatory drugs with the initial IVIG therapy.

Table 1. Clinical features of the four patients with coronary artery lesions after 30 days of illness.

\begin{tabular}{|c|c|c|c|c|c|c|}
\hline $\begin{array}{l}\text { No of } \\
\text { patients }\end{array}$ & Sex & $\begin{array}{l}\text { Age } \\
\text { of } \\
\text { onset }\end{array}$ & $\mathbf{S} / \mathbf{T}$ & $\begin{array}{l}1^{\text {st }} \text { IVIG } \\
\text { Response }\end{array}$ & $\begin{array}{l}2^{\text {nd }} \\
\text { IVIG }\end{array}$ & $\begin{array}{l}3^{\text {rd }}-\text { line } \\
\text { therapy }\end{array}$ \\
\hline 1 & Female & $2 \mathrm{y} 8 \mathrm{~m}$ & S & $\begin{array}{l}2 \mathrm{~g} / \mathrm{kg} / \text { day } \\
\text { for } 1 \text { day } \\
\text { resistant }\end{array}$ & yes & $\begin{array}{l}\text { plasma } \\
\text { exchange } \\
4^{\text {th }}-\text { line } \\
\text { therapy: } \\
\text { IVIG }\end{array}$ \\
\hline 2 & Male & $4 \mathrm{~m}$ & $\mathrm{~T}$ & $\begin{array}{l}2 \mathrm{~g} / \mathrm{kg} / \mathrm{day} \\
\text { for } 1 \text { day } \\
\text { resistant }\end{array}$ & yes & urinastatin \\
\hline 3 & Male & $11 \mathrm{~m}$ & $\mathrm{~T}$ & $\begin{array}{l}2 \mathrm{~g} / \mathrm{kg} / \text { day } \\
\text { for } 1 \text { day } \\
\text { resistant }\end{array}$ & yes & urinastatin \\
\hline 4 & Male & $1 \mathrm{y} 7 \mathrm{~m}$ & $\mathrm{~T}$ & $\begin{array}{l}2 \mathrm{~g} / \mathrm{kg} / \text { day } \\
\text { for } 1 \text { day } \\
\text { respond }\end{array}$ & no & no \\
\hline
\end{tabular}

No: number, IVIG: intravenous immunoglobulin,

S: S group, patients who received initial single IVIG therapy with delayed administration of anti-inflammatory drugs,

$\mathrm{T}$ : T group, patients who received concomitant anti-inflammatory drugs with IVIG,

y: year, m: month

Table 2 shows the background factors associated with the complications of CCAL and Egami scores of the patients [17]. Relapse, response after the initial IVIG therapy, and persistent fever after resistance of the initial IVIG therapy are the background factors.

Table 2. Background factors associated with the complications of coronary artery lesions after 30 days of illness and Egami score.

\begin{tabular}{lll}
\hline $\begin{array}{l}\text { No of } \\
\text { patients }\end{array}$ & Background factors & Egami score \\
\hline 1 & $\begin{array}{l}\text { Persistent fever after resistance of } \\
1^{\text {st }} \text { IVIG therapy }\end{array}$ & 4 \\
2 & Persistent fever after resistance of & 5 \\
3 & $1^{\text {st }}$ IVIG therapy & 3 \\
4 & Relapse & 2 \\
\hline
\end{tabular}

No: number, IVIG: intravenous immunoglobulin

Table 3. Sensitivity and specificity regarding initial IVIG therapy resistance and CAL complication.

\begin{tabular}{lll}
\hline & Sensitivity & Specificity \\
\hline IVIG resistance & $40 \%$ & $79 \%$ \\
CAL complication & & \\
CAL before day 30 & $60 \%$ & $77 \%$ \\
CAL after day 30 & $75 \%$ & $76 \%$ \\
\hline
\end{tabular}

IVIG: immunoglobulin therapy, CAL: coronary arterial lesions.

Table 3 shows the sensitivity and specificity regarding IVIG resistance and CAL complications as evaluated by the Egami score [17]. The Egami score could be retrospectively studied among all patients except for one who was an IVIGresponsive patient. As shown in Table 3, all sensitivities and 
specificities were less than $80 \%$.

\section{Discussion}

This study showed that the background factors for the development of CAL complications were variable and that an initial single IVIG therapy may be useful in the prevention of large CAL caused by these factors in the acute phase of Kawasaki disease. The establishment of a safe and effective regimen for initial IVIG therapy and the prevention of the development of large CAL during the acute phase are clinically important.

Recent Japanese guidelines for the medical treatment of acute Kawasaki disease recommended the use of risk scores that were developed to predict resistance to IVIG therapy; these guidelines also recommended the use of primary steroid therapy for those patients who were predicted to be IVIG-resistant [7]. However, a recent study showed that these risk-scoring systems had a low sensitivity for predicting IVIG resistance in a North American cohort [18]. Recently, other study in UK on Kawasaki disease also showed that the Kobayashi score did not predict IVIG resistance or CAL development in this population $[19,20]$. These findings were consistent with the results among Japanese patients (Table 3). Table 2 showed that IVIG resistance is associated with only half of the background factors associated with CCAL complications in patients who had received an initial $2 \mathrm{~g} / \mathrm{kg}$ IVIG therapy, and that relapse was another important factor associated with CCAL development.

One study about the combination of IVIG and steroids showed that large CAL were not prevented by this regimen [8]. One reason for the development of large CAL may be difficulty in administration of appropriate additional therapy because steroids modify the clinical course of Kawasaki disease.

Another study showed that a patient who had received initial IVIG and prednisolone combination therapy developed giant CAL after relapse [21]. This demonstrated the difficulties associated with administration of appropriate additional therapy after initial therapy with steroids. A single IVIG therapy does not modify the clinical course of Kawasaki disease. This characteristic permits clinicians to easily manage the treatment progress and to provide additional therapies at appropriate times during the clinical course. With these advantages and reported outcomes of CAL, initial single IVIG therapy may be superior to combination treatment with initial IVIG therapy and steroids.

A recent study found that primary steroid therapy did not improve coronary outcomes in patients who were prospectively classified as being high-risk for IVIG resistance [18]. The RAISE study also showed that primary steroid therapy could not prevent large CAL [8]. The results of the present study suggested the usefulness of an initial single IVIG therapy in the prevention of large CAL caused by Kawasaki disease. Recent research showed that antiinflammatory drugs including aspirin have a negative impact on the suppression of CAL development when administered with initial IVIG therapy during the acute phase of Kawasaki disease and that initial single IVIG therapy with delayed antiinflammatory drug administration may be beneficial in the suppression of CAL [11]. The results regarding the outcomes of CAL in this study were consistent with these findings.

Using logistic regression analysis, another study, which included patients who received IVIG therapy with and without delayed administration of anti-inflammatory drugs, showed that the significant variable for CAL development was the delayed administration of anti-inflammatory drugs and $2 \mathrm{~g} / \mathrm{kg} /$ day IVIG therapy and that the type of antiinflammatory drugs was not significant [11]. These findings were consistent with a recent comparative study regarding prevention of large CAL among four studies in which different four types of anti-inflammatory drugs were administered [4].

This study showed that the clinical courses and background factors associated with the formation of the CCAL complication were variable, and that the Egami score was not useful for predicting IVIG resistance and CCAL development. Therefore, the use of initial single IVIG therapy and appropriate additional therapy with critical clinical course observations may be useful in the prevention of large CAL caused by Kawasaki disease.

One limitation of this study was the small number of patients. In addition, this was a retrospective study. Finally, the use of the Japanese Ministry of Health and Welfare criteria may have underestimated the true incidence of CAL due to Kawasaki disease [22].

\section{Conclusions}

Variable factors including IVIG resistance, responsiveness, and relapse of the disease were associated with CAL complications. A single IVIG therapy does not modify the clinical course of Kawasaki disease. This characteristic permits clinicians to easily manage the treatment progress and to provide additional therapies at appropriate times during the clinical course. An initial single IVIG therapy may be useful in the prevention of large CAL caused by different factors of Kawasaki disease.

\section{Acknowledgements}

I would like to thank the pediatric cardiologists of Hirosaki University School of Medicine for providing clinical information about patient 1 , all those who were involved in the medical management of the patients included in this study, and Enago (www.enago.jp) for the English language review.

\section{References}

[1] Burns JC, Glodé MP. Kawasaki syndrome. Lancet 2004; 364:533-544. 
[2] Muller F, Knirsch W, Harpes P, Prêtre R, Valsangiacomo BE, Kretschmar O. Long-term follow-up of acute changes in coronary artery diameter caused by Kawasaki disease: risk factors for development of stenotic lesions. Clin Res Cardiol 2009; 98:501-507.

[3] Tsuda E, Kamiya T, Ono Y, Kimura K, Kurosaki K, Echigo S. Incidence of stenotic lesions predicted by acute phase changes in coronary arterial diameter during Kawasaki disease. Pediatr Cardiol 2005; 26:73-79.

[4] Nakada T. Prevention of large coronary artery lesions caused by Kawasaki disease. Medical Research Archives 2015. DOI: http://dx.doi.org/10.18103/mra.v0i3.138

[5] Furusho K, Kamiya T, Nakano H, et al. High-dose intravenous gammaglobulin for Kawasaki disease. Lancet 1984; 2: 1055 1058 .

[6] Newburger JW, Takahashi M, Beiser AS, et al. Single intravenous infusion of gamma globulin as compared with four infusion in the treatment of acute Kawasaki syndrome. N Engl J Med 1991; 324:1633-1639.

[7] Research committee of the Japanese Scociety of Pediatric Cardiology; Cardiac Surgery committee for development of guidelines for medical treatment of acute Kawasaki disease. Guidelines for medical treatment of acute Kawasaki disease: report of the Research committee of the Japanese Society of Pediatric Cardiology and Cardiac Surgery (2012 revised version). Pediatr Int 2014; 56:135-158.

[8] Kobayashi T, Saji T, Otani T, et al. Efficacy of immunoglobulin plus prednisolone for prevention of coronary artery abnormalities in severe Kawasaki disease (RAISE study): a randomized, open-label, blinded-endpoints trial. Lancet 2012; 379:1613-1620.

[9] Tremoulet $\mathrm{AH}$, Jain $\mathrm{S}$, Jaggi $\mathrm{P}$, et al. Infliximab for intensification of primary therapy for Kawasaki disease: a phase 3 randomised, double-blind, placebo-controlled trial. Lancet 2014; 383:1731-1738.

[10] Uehara R, Belay ED, Maddox RA, et al. Analysis of potential risk factors associated with nonresponse to initial intravenous immunoglobulin treatment among Kawasaki disease patients in Japan. Pediatr Infect Dis J 2008; 27:155-160.

[11] Nakada T. Effects of anti-inflammatory drugs on intravenous immunoglobulin therapy in the acute phase of Kawasaki disease. Pediatr Cardiol 2015; 36:335-339.

[12] Nakada T. Study on the patients with Kawasaki disease associated with coronary artery lesions before therapy. Japanese Journal of Pediatrics 2015; 68:1410-1414.

[13] Nakamura Y, Yanagawa H, Ojima T, Kawasaki T, Kato H. Cardiac sequelae of Kawasaki disease among recurrent cases. Arch Dis Child 1998; 78:163-165.

[14] Yang H, Du ZD, Fu P. Clinical features of recurrent Kawasaki disease and its risk factors. Eur J Pediatr 2013; 172:16411647.

[15] The Japan Kawasaki disease research committee. Diagnostic guidelines of Kawasaki disease. 5th revised ed, Tokyo, 2002.

[16] Research committee on Kawasaki disease. Report of subcommittee on standardization of diagnostic criteria and reporting of coronary artery lesions in Kawasaki disease. Ministry of Health and Welfare, Tokyo, 1994.

[17] Egami K, Muta H, Ishii M, et al. Prediction of resistance to intravenous immunoglobulin treatment in patients with Kawasaki disease. J Pediatr 2006; 149:237-240.

[18] Sleeper LA, Minich LL, McCrindle BM, et al. Evaluation of Kawasaki disease risk-scoring systems for intravenous immunoglobulin resistance. J Pediatr 2011; 158:831-835.

[19] Davies S, Sutton N, Blackstock S, et al. Predicting IVIG resistance in UK Kawasaki disease. Arch Dis Child 2015; 100:366-368.

[20] Kobayashi T, Inoue Y, Takeuchi K, et al. Prediction of intravenous immunoglobulin unresponsiveness in patients with Kawasaki disease. Circulation 2006; 113:2606-2612.

[21] Ashida A, Ozaki N, Kishi K, Katayama H, Okasora K, Tamai H. A case report of refractory Kawasaki disease with bilateral giant coronary aneurysms treated with intravenous immunoglobulin and prednisolone combination therapy. Progress in Medicine 2013; 33:1471-1474.

[22] Zorzi AD, Colan SD, Gauvreau K, Baker AL, Sundel RP, Newburger JW. Coronary artery dimensions may be misclassified as normal in Kawasaki disease. J Pediatr 1998; $133: 254-258$ 